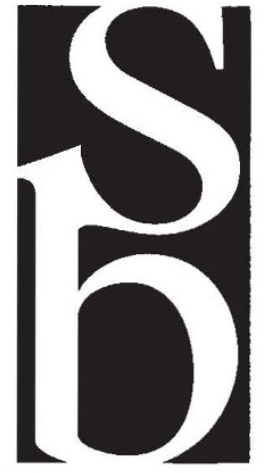

\section{Structural Biology in Chicago}

The job description 'I'm a structural biologist' (not recommended at most parties) is becoming more and more meaningless as the emphasis in the field shifts firmly from structure to biology. Structural biology, like molecular biology, is no more than a loose amalgam of technologies (albeit sophisticated ones) providing researchers with a range of tools with which to analyze the structure of their chosen molecule(s). This was ably demonstrated by the presentations at the first annual Nature Structural Biology conference* which fully illusrated the degree to which biology now dominates structural biology.

"If you want to understand function, study structure": the quote, from Francis Crick, framed the opening talk from Paul Sigler (HHMI, Yale University). Sigler also touched on biology's 'Dark Matter' problem, that is, high resolution structural techniques, such as X-ray crystallography and nuclear magnetic resonance (NMR), can only'see' orderly structures whereas the very nature of many, if not most, macromolecules is one of motion and disorder-states which are at present difficult to analyze in detail. Even so, Sigler is using established 'tricks of the trade' to characterize the action of the small G-protein transducin on the signal transduction pathway in the visual system of vertebrates.

The Dark Matter problem is nowhere more apparent than in the study molecular motors. One of the latest myosin head structures (this time from Dictyostelium), reported by Ivan Rayment (University of Wisconsin, Madison), shows a conformational change that results in what is effectively a $20^{\circ}$ rotation of the $\mathrm{C}$ terminus of the molecule. Due to the length of the domain, this translates into an overall movement at the end of the molecule of 20-30 . Clearly such a conformational change is at the heart of the motor's function and must be in some way coupled to the

*Structural Biology: The Molecules of Life, Chicago, Illinois, 2-4 April 1995. protein's power source; the hydrolysis of ATP. Robert Fletterick (University of California, San Francisco) provided the first tantalizing glimpse of the structure of the motor kinesin.

RNA, Jamie Williamson (Massachusetts Institute of Technology) noted, has an informational, a structural, a catalytic and a regulator role in cells. Nevertheless, there are no more than a handful of RNA or RNA/protein structures presently in the literature. The binding of the HIV REV protein to the RRE plays a critical role in the life cycle of the virus. Williamson's analysis of the nature of the interaction between these important regulatory elements may play a vital role in the war against AIDS. The structure of a catalytic RNA, the hammerhead ribozyme, was presented by David McKay (Stanford University). The catalytic core is comprised of two domains. Domain I is similar to the uridine turn found in tRNA and domain II consists of a stack of three non-Watson and Crick base pairs and a tandem G:A mismatch which binds divalent ions. The structure of the ribozyme/ inhibitor complex seems to represent the ground state of the enzyme, as there are no functional groups in a position to attack the phosphodiester backbone. The challenge now is to characterise the conformational states that lead to catalysis.

Teleomeres are essential for the complete replication of the ends of eukaryotic chromosomes and are thought to play a critical role in determining the correct association between homologous chromosomes. Daniela Rhodes (MRC,Laboratory of Molecular Biology) reported on studies of the RAP1 protein and its interaction with telomeres. The simple repeated sequences at the ends of chromosomes are thought to form four-stranded G-tetrad structures, the only problem being that in vitro, their formation requires high concentrations of DNA and is very slow. The RAP1 protein seems to present a way around this problem: the protein not only binds to the GTstrand of yeast telomeric sequences but seems to promote the formation of G-tetrads, further indirect evidence that telomeres do consist of Gtetrads. The toriodal structure of another DNAbinding protein, Escherichia coli Topoisomerses I, 
allowed Alfonso Mondragon (Northwestern University) to present an elegant model of how this enzyme goes about changing the topology of double-stranded DNA.

The TATA-box binding protein (TBP) plays a central role in the formation of the transcription initiation complexes of all three nuclear RNA polymerases, as reported by Stephen Burley (HHMI, Rockefeller University). Because of the long half life of the TBP/DNA complex, TBP remains stably bound to the distorted promoter DNA after the elongation factors have moved off down the DNA allowing rapid reinitiation of the transcription complex without the penalty of having to reassemble the complex on the DNA. Thus transcriptional efficiency may be modulated by a change in the $t_{1 / 2}$ of the TBP/ DNA complex. The 'portable' molecular dimerization system described by Stuart Schreiber (HHMI,Harvard University) provides a useful new tool (a kind of 'chemical genetics') for probing the molecular events that function though proximity, such as those of gene transcription. Molecular chaperones mediate the conversion of nascent polypeptide chain or unfolded protein to the fully folded state. Arthur Horwich (HHMI, Yale University) has used the recently determined structure of the chaperonin GroEL to direct mutagenesis studies. He finds, for example, that the polypeptide- and GroES-binding sites thus defined overlay each other and consist mainly of hydrophobic residues on the inside surface of the apical domain. Thus (along with the binding of ATP to GroEL) one function of the smaller GroES ring may be to displace the bound, partially folded, polypeptide from the inside apical surface into the cavity in the GroEL doughnut. Cycles of binding and dissociation are then thought to result in productive folding.

Another large molecular machine that has yielded to crystallographic analysis is $\mathrm{F}_{1}$-ATPase, the enzyme responsible for generating ATP from $A D P$ and phosphate. Andrew Leslie (MRC, Laboratory of Molecular Biology) presented video footage of a proposed rotational catalytic mechanism for the multisubunit, mushroom-shaped enzyme. The globular 'head' and long $\alpha$-helical 'stalk' of the mushroom rotate relative to one another, under the influence of the protonmotive force across chloroplast, mitochondrial and bacterial membranes. The hydrophobicC-terminal stalksits in a greasy sleeve in the head domain, looking very much like a molecular bearing. The curved stalk acts like a cam in the model, influencing the conformation of the $\beta$-subunits in the head and hence, presumably, driving the formation of ATP)

Stephen Harrison (HHMI, Harvard University) noted that for non-enveloped viruses, such as polyomervirus, it is not clear how the virus manages to traverse the physical as well as topological barrier of the cell membrane. A first step along this path is the characterization the initial interaction of polyomervirus with its carbohydrate ligand. Such studies show that the specificity of the interaction is of vital importance in determining the viruses ability to establish a disseminated infection and to induce tumours in the host.

The heart of the photosynthetic machinery is slowly being revealed by the probings of structural biologists and biophysicists. Leslie Dutton (University of Pennsylvania) presented a detailed discussion of the nature of long-range electron transfer processes in proteins, with particular reference to the photosynthetic reaction centre (RC) while Hartmut Michel (Max-Planck-Institit fur Biophysik) presented a description of the high resolution structure of the Rhodobacter sphaeroides RC. The prokaryotic enzyme photolyase also uses light, but to a different end: repair of thymindine dimers $(\mathrm{T}=\mathrm{T})$ in damaged DNA. Johann Deisenhofer (HHMI, University of Texas Southwestern Medical Centre) described how the structure of the protein reveals a molecular surface that may bind DNA and facilitate insertion of $\mathrm{T}=\mathrm{T}$ into what is most likely the active-site hole, which bears a complementary arrangement of chemical groups. Here the bases are separated. machines that direct the processes of life are firmly in the sights of the structural biologists and biologists concerned with structure: how could they resist the challenge?
The multicomponent

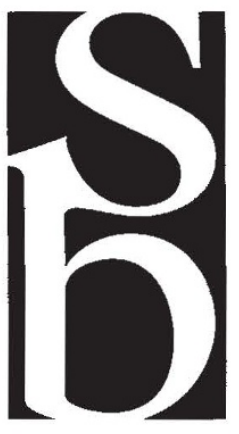

structural biology volume 2 number 5 may 1995 